\title{
A Study on The Appearance of Spatial Illuminance of The Lampshade Using The Folds of Linear Geometric Pattern
}

\author{
Yoshie Takewakaa, ${ }^{\mathrm{a},}$, Masanobu Inoue ${ }^{\mathrm{a}}$, Harunobu Kuwano ${ }^{\mathrm{a}}$, Makoto Miyauchi ${ }^{\mathrm{a}}$, Seiichi Serikawa ${ }^{\mathrm{b}}$ \\ ${ }^{a}$ National Institute of Technology, Kitakyushu College, 5-20-1 Shii Kokuraminami Kitakyushu, Fukuoka 802-0985, \\ Japan \\ ${ }^{b}$ Kyushu Institute of Technology, 1-1 Sensui Tobata Kitakyushu, Fukuoka, 804-8550, Japan \\ *Corresponding Author: yoshie @kct.ac.jp
}

\begin{abstract}
The history of the light is the history of lighting equipment, and the shapes of the shade changed with the change of the light source. In this report, we are going to prove that the value of spatial illuminance varies by the variation and combination of the folds of linear geometric pattern on shoji paper, and that the impression given by the shadow of the lampshade changes with the variation of the shadow of the lampshade.
\end{abstract}

Keywords: lampshade, spatial illuminance, linear geometric pattern.

\section{Introduction}

The history of the light is the history of lighting equipment, and the shapes of the shade changed with the change of the light source. From the Middle Ages to the Modern Times, because burning light source (candles, oil, gas and so on) were used as lighting both indoors and outdoors, lamp shades were designed to avoid wind and rain such as hand lamps and lanterns. Later, the light source changed to the one (arc lamp, incandescent electric bulb, fluorescent lamp, LED) which uses electrical power, and that change made lighting equipment brighter and more energy-saved. Due to this change, in the outdoor illumination the lampshades come to be made in order to avoid rain than wind, and in the indoor one the designs of the lampshade come to be thought more important than their brightness.

In current lampshades, various materials are used, such as paper, cloth glass, ceramics, and the plastics and so on. Particularly as to paper and cloth, various people from amateurs to professionals produce various lampshades by these materials and their works are exposed on various website.

Furthermore, Suzuki and others presented that it is able to design a lampshade by using a ruled surface by controlling the parameter of the ruled surface because of the continuity of normal vectors and that it is able to structure lampshade from some ruled surfaces by using the system of an additive manufacturing to the frame part of modeled object. Suzuki also introduced his attempt in which students manufactured paper models of curved surface-shaped lampshades by using three-dimensional computer graphics in graphic science education. ${ }^{(1)-(4)}$

In this report, we are going to prove that the value of spatial illuminance varies by the variation and combination of the folds of linear geometric pattern on shoji paper, and that the impression given by the shadow of the lampshade changes with the variation of the shadow of the lampshade.

\section{Experiment and Discussion}

\subsection{Preliminary experiment}

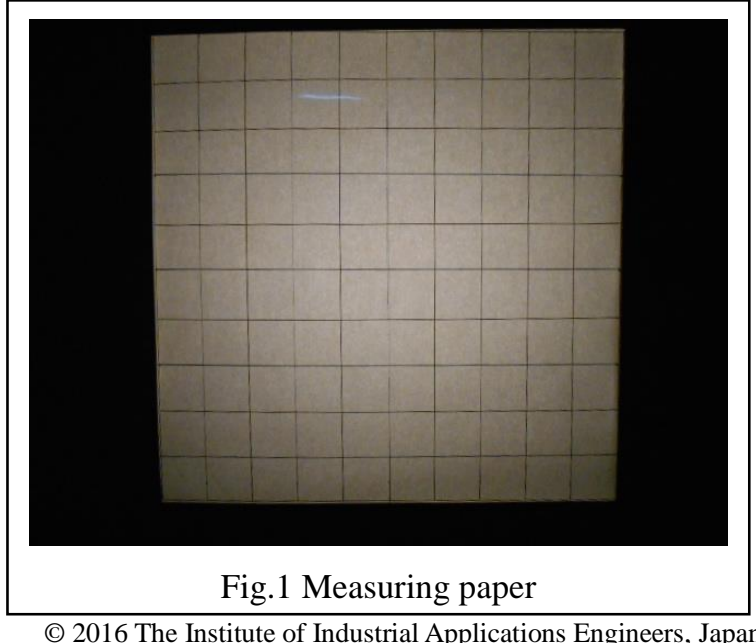


In this experiment, we used as the light source an

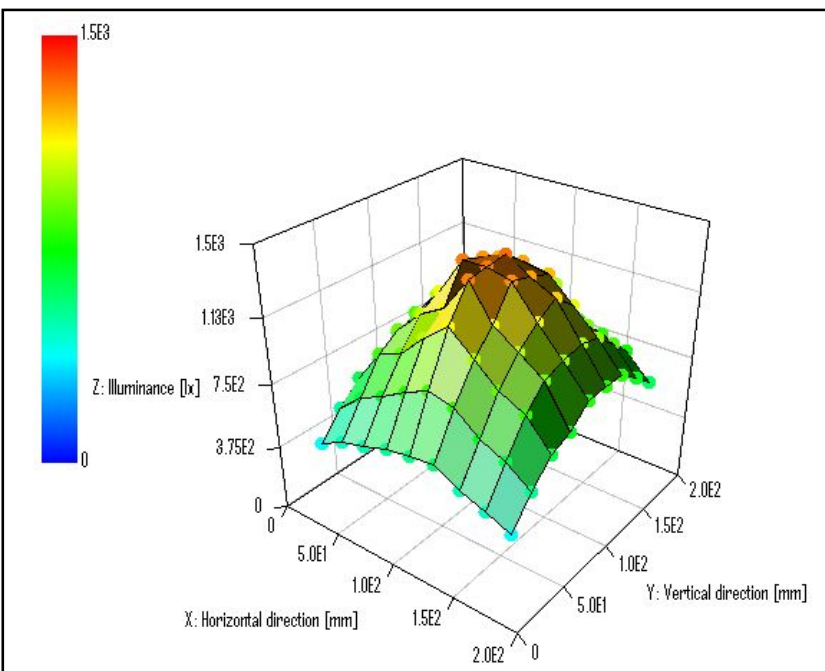

(a) The result of one sheet of shoji paper

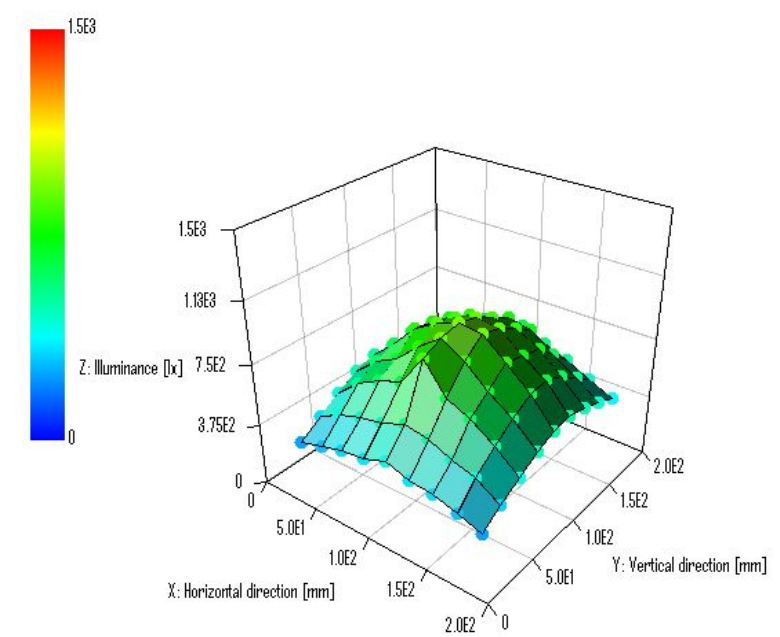

(b) The result of shoji paper two sheets

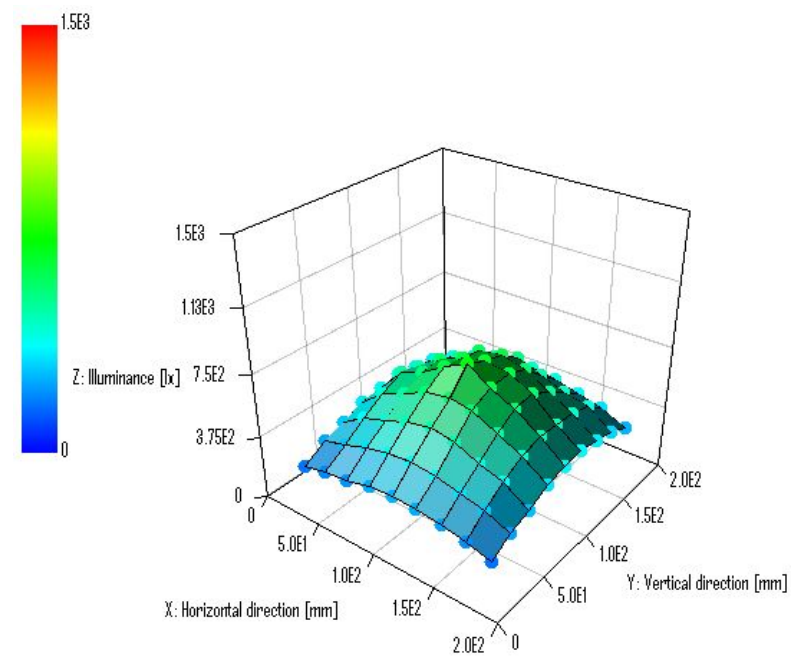

(b) The result of shoji paper three sheets

Fig.2 Distance from the LED light bulb 100 [mm] measurement results electric bulb type LED lamp made in Toshiba (LDR8L-G/60W: total luminous flux 810[1m], power consumption 7.8[W], energy consumption efficiency $103.8[1 \mathrm{~m} / \mathrm{W}]$, omni-directional type with the expanse 260 degrees of the light).

To decide the optimum position of the lampshade, we performed following preliminary experiment: we put one sheet of shoji paper on the frame of a cardboard shown in Fig. 1 and we set the measuring range of $200 \times 200$ [mm], and assumed the lattice point of the interval $20[\mathrm{~mm}]$ as the measuring point. We measured spatial illuminances with the illuminometer (HIOKI3422) where the horizontal distances from the center of the electric bulb was $50[\mathrm{~mm}], 100[\mathrm{~mm}]$, and $150[\mathrm{~mm}]$ respectively. In addition, conditions of the laboratory were temperature $26.6\left[{ }^{\circ} \mathrm{C}\right]$, humidity $51.0[\%]$, illuminance (disturbance) $5.70[1 \mathrm{x}]$ of the measuring point. We performed that experiment under this condition.

The results of these measuring are as follows: In the case of one sheet of shoji paper, the maximum illuminance and the minimum illuminance were $1340[1 \mathrm{x}]$ and 385[1x] respectively. In the case of two sheets of shoji paper, the maximum illuminance and the minimum illuminance were $926[1 x]$ and $243[1 x]$ respectively. In the case of three sheets of shoji paper, the maximum illuminance and the minimum illuminance were $730[1 \mathrm{x}]$ and $172[1 \mathrm{x}]$ respectively. We show the result in Fig.2, using 3-dimensional graph-making application (RINEARN Graph3D 5.5).

From Fig.2(a), (b), (c), it became clear that the center of the measuring paper was the position of maximum illuminance of the LED bulb and both ends of the measuring paper were the position of minimum illuminance because light does not spread through lower part 100 degrees of the bulb. And we decided to choose those lattice points as measuring points. Here, we adopt gradation

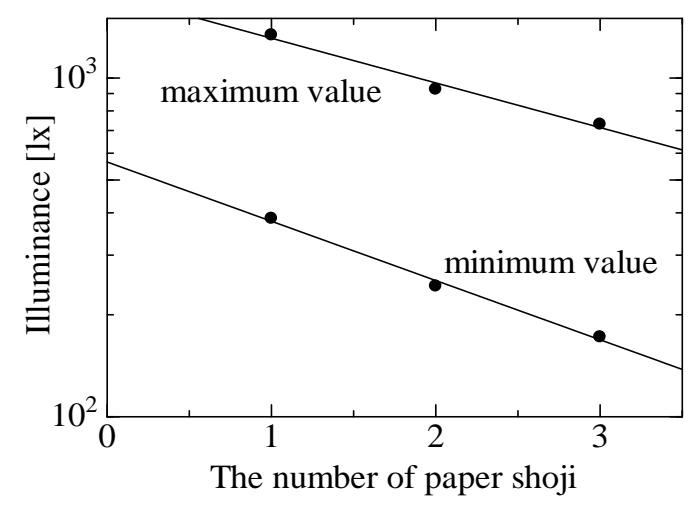

Fig.3 Attenuation approximate value by the number of sheets of paper shoji 
display in Fig.2 to make the contrast clear. The setting of the color of each point is as follows: the point where its illuminance is near to $0.00[1 \mathrm{x}]$ is bluer and the point where its illuminance is near to $1500[1 \mathrm{x}]$ is redder. The appearance of the gradation is shown in the left bar.

We show the maximum value and the minimum value of the spatial illuminance in Fig. 3 by a graph. The vertical axis indicates with logarithmic scale and the horizontal axis indicates the number of sheets of shoji paper. We understand from this graph that spatial illuminance decreases logarithmically because values at the measuring points are enabled collinear approximation.

\subsection{Experiment and Discussion}

In order to make the folds of linear geometric pattern, we folded shoji paper with the mountain fold and the valley fold alternately, and then we gathered one of the ends in a vertex and spread the other end like a fan and we folded the peaks of the mountain folds down to one side. We made

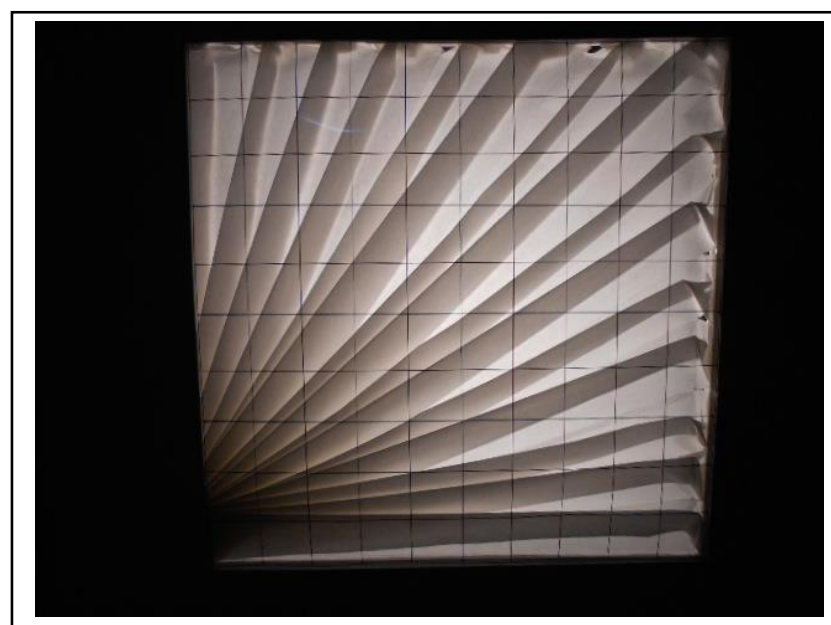

(a) Mounting the end face on the lower left

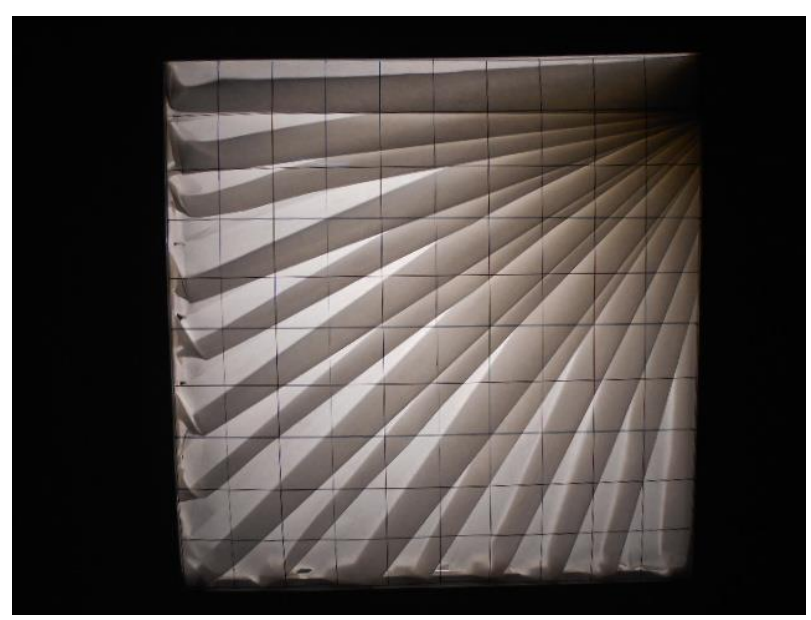

(b) Mounting the end face on the upper right corner Fig.4 Folding width 20 [mm] two types with $20[\mathrm{~mm}]$ width and $30[\mathrm{~mm}]$ width between the mountain fold and the valley fold.

In Fig.4 and 5, we show the appearance of shadow through the folds when the LED bulb was turned on. Both (a) and (b) of Fig.4 and Fig.5 describe respectively in the case that the gathered end was mounted at the bottom-left corner and at the upper-right corner. Since the LED bulb was omni-directional type with the expanse 260 degrees of the light, we put the folds as (a) and (b) and then we checked its appearances.

After that, we set the measuring range of $200 \times 200[\mathrm{~mm}]$ and choose the lattice point of the interval $20[\mathrm{~mm}]$ as the measuring point and measured spatial illuminances with the illuminometer (HIOKI3422) similarly as the preliminary experiment. In addition, conditions of the laboratory, which were temperature $26.6\left[{ }^{\circ} \mathrm{C}\right]$, humidity $51.0[\%]$, and illuminance (disturbance) $5.70[1 \mathrm{x}]$ of the measuring point were the same as the preliminary experiment.

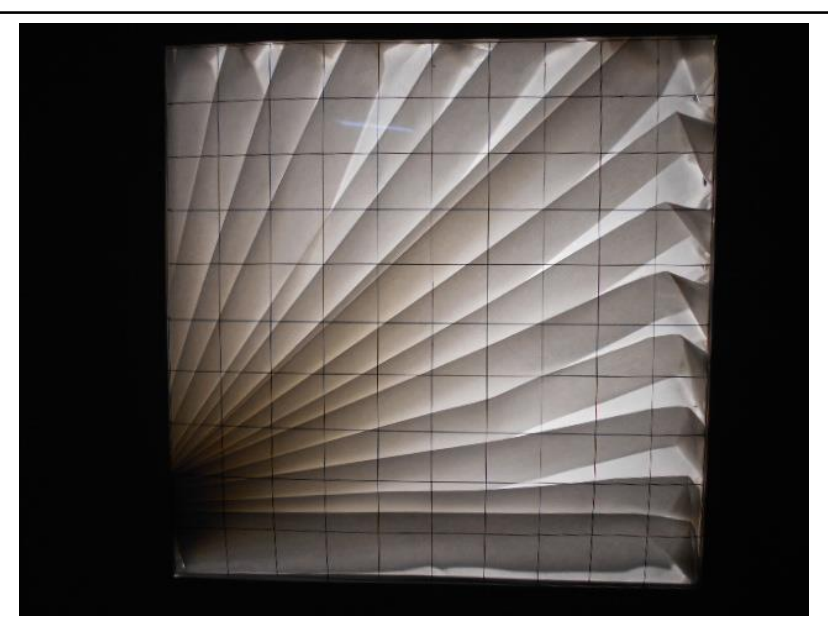

(a) Mounting the end face on the lower left

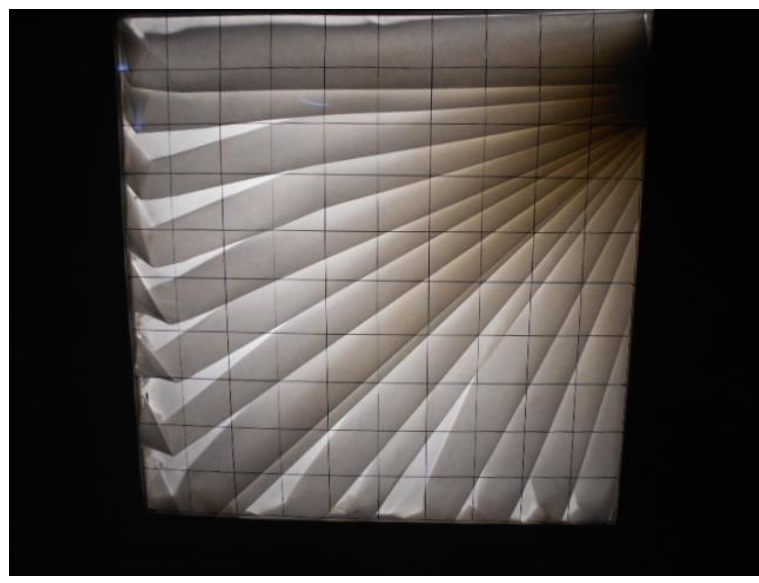

(b) Mounting the end face on the upper right corner Fig.5 Folding width 30 [mm] 
Here, we adopt gradation display in Fig.6 and Fig.7 to make the contrast clear. The setting of the color of each point is as follows: the point where its illuminance is near to $0.00[1 \mathrm{x}]$ is bluer and the point where its illuminance is near to $1500[1 \mathrm{x}]$ is redder. The appearance of the gradation is shown in the left bar.

From Fig.4, we can confirm that the area of the overlapping part of the folds more distant from the gathered end gets smaller. In other words, we can confirm that the transmitted light of the LED bulb increased, and the value of the spatial illuminance rose. In the case described in Fig.6(a), the maximum illuminance was $918[1 \mathrm{x}]$ at the same position as the preliminary experiment of using two sheets of shoji paper, and the minimum illuminance was $68[\mathrm{x}]$ at

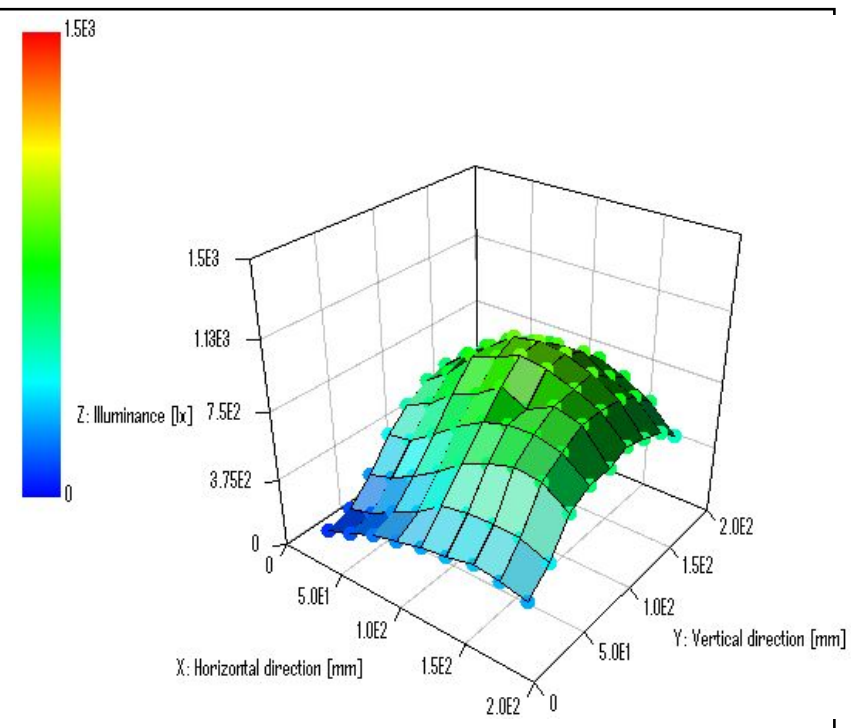

(a) Mounting the end face on the lower left

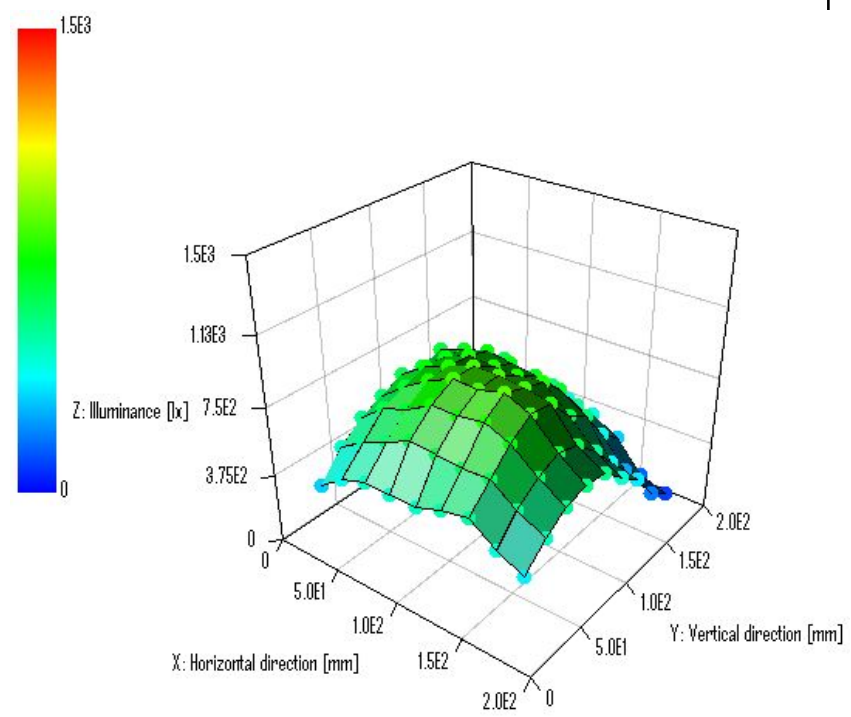

(b) Mounting the end face on the upper right corner

Fig.6 Folding width 20 [mm] the bottom-left corner. In the case described in Fig.6 (b), the maximum illuminance was $845[1 \mathrm{x}]$ at the position $40[\mathrm{~mm}]$ lower than (a)'s maximal position, and the minimum illuminance was $93[\mathrm{~lx}]$ at the upper-right corner.

From Fig.5, we can also confirm that the area of the overlapping part of the folds more distant from the gathered end gets smaller, but the value of spatial illuminance is smaller than in the case of Fig.6 because most part of measuring range is overlapping. We show it in Fig.7.

In the case described in Fig.7 (a), the maximum illuminance was $598[1 \mathrm{x}]$ at the position $40[\mathrm{~mm}]$ upper than the maximal position in Fig.6, and the minimum illuminance was $65[1 \mathrm{x}]$ at the bottom-left corner. In the case described figure $7(\mathrm{~b})$, the maximum illuminance was

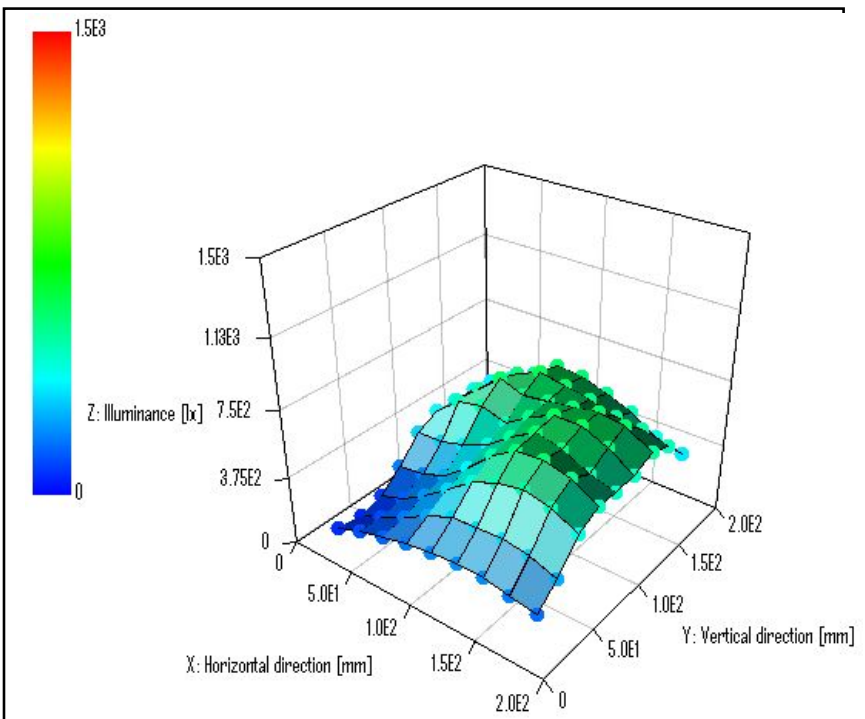

(a) Mounting the end face on the lower left

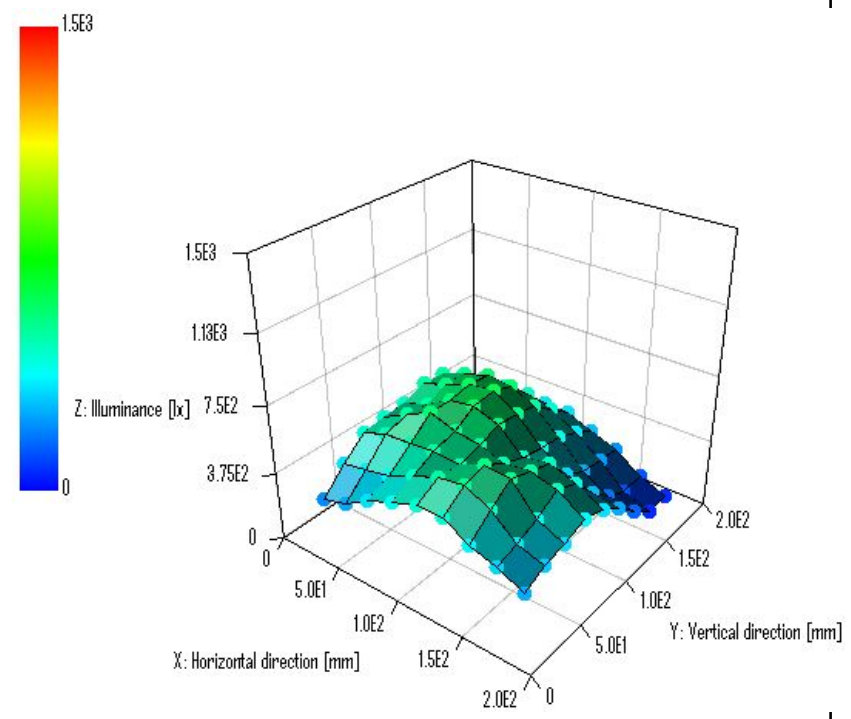

(b) Mounting the end face on the upper right corner

Fig.7 Folding width $30[\mathrm{~mm}]$ 
$562[\mathrm{~lx}]$ at the position $60[\mathrm{~mm}]$ upper than (a)'s maximal position, and the minimum illuminance was $61[1 \mathrm{x}]$ at the upper-right corner. Furthermore, four sheets of shoji paper are overlapping along the diagonal line from the gathered end because two folds are overlapping in Fig.5. Then the graph of spatial illuminance in Fig.7 has a different form from one in Fig. 6 and we can confirm that it has dark zone on the diagonal line.

Thus, we can prove that the value of spatial illuminance varies by the variation and combination of the folds of linear geometric pattern, and that the impression given by the shadow of the lampshade changes with the variation of the shadow of the lampshade.

\section{Conclusions}

We have proved that the value of spatial illuminance varies by the variation and combination of the folds of linear geometric pattern on shoji paper, and that the impression given by the shadow of the lampshade changes with the variation of the shadow of the lampshade.

We have also proved that the spatial illuminance of transmitted light through shoji paper decreases logarithmically in the preliminary experiment.

We are planning to make a different shadow in consideration of various geometric patterns and introduce new lampshade. Furthermore, we are also planning to produce shaping of not only planar lampshade but also three-dimensional one.

\section{References}

(1) Hirotaka Suzuki: "Design of Lamp Shade that Uses Ruled Surface - Approach of Lamp Shade Design by Accumulating Forming System - "Journal of Graphic Science of Japan, Vol. 42, No. Supplement2, pp. 63-66, 2008.

(2) Hirotaka Suzuki: "Designing of the lamp shade by tangent surface", Journal of Graphic Science of Japan, Vol. 44, No. 3, pp. 15-16, 2010.

(3) Hirotaka Suzuki, Ai Sakaki, Kensuke Yasufuku and Takashi Matsumoto: "Designing of lampshade with 3D CG application and manufacturing of designed shape in graphic science education", Int. J. Computer Applications in Technology Vol. 51, No. 1, pp. 9-14, 2015.

(4) Hirotaka Suzuki: "A Study on Impact of Introduction of Lighting Equipment Design Assignment into Graphic Science Education", Proc. of The 12th International Conference on Geometry and Graphics(2006), \#E 17 (CD-ROM) 